Y.-H. Chu, N.B. Suntzeff, J.E. Hesser, and D.A. Bohlender, eds.

\title{
Bow Shock Induced Star Formation in the LMC
}

\author{
K.S. de Boer, J.M. Braun \\ Sternwarte, University of Bonn, D-53121 Bonn, Germany
}

\author{
A. Vallenari \\ Osservatorio Astronomico, Vic. Osserv. 5, I-35122 Padova, Italy
}

U. Mebold

Radioastronomisches Institut, Univ. Bonn, D-53121 Bonn, Germany

\section{Facts}

The supergiant shell LMC 4 is known for its ring of $\mathrm{H}$ II regions and the deep minimum in $\mathrm{HI}$ in the interior (Rohlfs et al.1984). LMC 4 shows X-ray emission detected by ROSAT and highly ionised gas (C IV) detected with HST (Bomans et al. 1994, 1996). The velocities of its neutral gas indicate it cannot have been formed by the collision with a high velocity cloud (Domgörgen et al. 1995).

The age of the association NGC 1948 at the NE edge of LMC 4 (Vallenari et al. 1993, Will et al.1996) is 5-10 Myr, while the stars in the interior are 10-15 Myr old (Braun et al. 1997). The similarity of all the ages derived for star groups inside and at the edge of LMC 4 (Braun et al.1997) pointed toward the need to identify a large scale star formation triggering event.

ROSAT X-ray data of the LMC were analysed by Blondiau et al. (1997) and they found near the dark cloud south of 30 Dor an anticorrelation between $\mathrm{X}$-ray emission and neutral hydrogen + IRAS dust emission. They noted also that this region is close to and aligned with the steep gradient in $\mathrm{H}$ I near the leading edge, a region from which the $\mathrm{X}$-ray intensity is lower than the general background of the X-ray sky (see map of Snowden \& Petre 1994).

\section{Model}

Collectively, the described facts led to the following scenario. Gas is compressed at the leading edge of the LMC because of the bow shock of the LMC formed in the interaction with the galactic halo. Such compressed gas starts to form stars, likely on a large scale. Due to the rotation of the LMC these regions move away from the leading edge, and a sequence of such events will lead to a sequence of structures along the eastern and northern edge on the LMC increasing in age in a clockwise fashion (de Boer et al.1998).

The kinematics of the LMC are as follows. Models for the Magellanic Clouds and Stream (Heller \& Rohlfs 1994) indicate that the LMC moves towards the East, confirmed by Hipparcos data of LMC stars (Kroupa \& Bastian 1997). The full space velocity is $\simeq 265 \mathrm{~km} \mathrm{~s}^{-1}$. Combining the radial and the tangential 
rotation curve, the true rotation speed of the LMC at a radius of $2 \mathrm{kpc}$ is $\simeq 150$ $\mathrm{km} \mathrm{s}^{-1}$. Given the above, the maximum velocity difference between LMC gas and gas of the Milky Way halo is $\simeq 450 \mathrm{~km} \mathrm{~s}^{-1}$, to be found near the SE edge.

\section{Predictions}

The clockwise progression of age, starting at the leading edge as given by the fields listed by de Boer et al.(1998), should continue along the edge of the LMC and populations older than $50 \mathrm{Myr}$ should be found at the western and southern edges. The star formation at the leading edge of the LMC need not be a continuous process. Interruptions in the piling up of gas may occur when a region devoid of gas is moving up to the leading edge, such as a 'former LMC 4'. The NE side of the LMC shows such gaps.

The bow shock induced star formation model predicts a clock-wise increase in the age of superstructures, in step with the rotation velocity of $\simeq 150 \mathrm{~km} \mathrm{~s}^{-1}$. At a radius of about $2 \mathrm{kpc}$ this means one revolution in $\simeq 100 \mathrm{Myr}$. One might therefore expect at each location along the outer edge of the LMC stellar populations with ages of modulo $100 \mathrm{Myr}$. Looking for the sequence of ages, only accurate ages are of use, with precision better than $20 \%$. Such a precision can be reached with star groups, but hardly when using individual objects. Other mechanisms of star formation also operate in the LMC (de Boer et al. 1998), and hence the basic bow shock structure may be overlain with secondary star formation and thus with other ages.

The inclination of the LMC with respect to the line of sight implies that the vector of motion of the LMC lies in the plane of the LMC itself. Thus the LMC slices its way as a disk through the MW halo. The radial rotation curve of the LMC indicates solid body rotation out to $2 \mathrm{kpc}$. This means that the geometry of the star forming regions near the leading edge may stay the same during the further rotation. Is it significant that the shape of the dark cloud south of 30 Dor is the same as that of association LH 77 inside LMC 4?

\section{References}

Blondiau, M.J., Kerp, J., Mebold, U., \& Klein, U. 1997, A\&A, 323, 585

Bomans, D.J., Dennerl, K., \& Kürster, M. 1994, A\&A, 283, L21

Bomans, D.J., de Boer, K.S., Koornneef, J., et al. 1996, A\&A, 313, 101

Braun, J.M., Bomans, D.J., Will, J.-M., \& de Boer, K.S. 1997, A\&A, 328, 167 de Boer, K.S., Braun, J.M., Vallenari, A., \& Mebold, M. 1998, A\&A, 329, L49 Domgörgen, H., Bomans, D.J., \& de Boer, K.S. 1995, A\&A, 296, 523

Heller, P., \& Rohlfs, K. 1994, A\&A, 291, 743

Kroupa, P., \& Bastian, U. 1997, New Astronomy, 2, 77

Rohlfs, K., Kreitschmann, J., Siegman, B.C., et al. 1984, A\&A, 137, 343

Snowden, S.L., \& Petre, P. 1994, ApJ, 436, L123

Vallenari, A., Bomans, D.J., \& de Boer, K.S. 1993, A\&A, 268, 137

Will J.-M., et al. 1996, A\&A, 315, 125 